EPJ Web of Conferences 65, 01001 (2014)

DOI: 10.1051/epjconf/20146501001

(C) Owned by the authors, published by EDP Sciences, 2014

\title{
Crystal scintillators for low background measurements
}

\author{
R. Bernabei ${ }^{1}$ \\ ${ }^{1}$ Dipartimento di Fisica, Universita' di Roma Tor Vergata, I-00133 Rome, Italy and INFN, Sezione di Roma Tor Vergata, I-00133 \\ Rome, Italy
}

\begin{abstract}
.
Some general arguments in the light of the application of the inorganic crystal scintillators in the search for rare processes are addressed. The continuous innovation provided by physics, chemistry and technology in their development allows the improvement of their performances. Also the possibility of detectors exploiting new materials is open and the further application to this field of research is promising.
\end{abstract}

\section{Introduction}

The development of inorganic crystal scintillators regards a field where physics, chemistry and technology produce a continuous innovation for the applications in many research fields. A number of new crystal scintillators has been developed, while even the earliest inorganic scintillators are still competitive due to the renewed technology. When the radio-purity of such detectors is an additional requirement, their application in the investigation of rare processes becomes competitive. Moreover, the possibility of significant enrichments offers additional relevant tools for the investigation of several rare processes such as: Dark Matter candidates of various nature, $\beta \beta$ decay modes, rare nuclear decay modes, solar axions, electron stability, matter stability, search for exotics in cosmic rays, etc.

The physical characteristics (high detection efficiency, physical shape, chemical and mechanical stability) and the luminescent characteristics (emission wavelength, high light yield, good linearity, suitable decay time and in some application good radiation hardness) of the best qualities inorganic scintillators have been frequently underlined.

The strengths of experiments with low-background crystal scintillators are: i) well known technology; ii) high duty cycle; iii) large mass possible; iv) enrichment possible e.g. for $\beta \beta$ decays investigations; v) ecologically clean set-up; no safety problems; vi) cheaper than other considered technique; vii) relatively small underground space needed; viii) high radio-purity by selections, chem./phys. purifications, protocols reachable; ix) well controlled operational condition feasible; $\mathrm{x}$ ) neither re-purification procedures nor cooling down/warming up (reproducibility, stability, ...); xi) possibility of high light response in many cases; xii) effective routine calibrations feasible in the same conditions as production runs; xiii) absence of microphonic noise; xiv) possibility of application both in passive and active source approaches as well as with coinci- dence/anticoincidence techniques; $\mathrm{xv}$ ) many isotopes and decay modes explorable; $\mathrm{xvi}$ ) etc.

In particular, in the field of rare processes the high radio-purity, the stability of performances, the possible choice of different isotopes, and the high light yield have allowed the detectors to increase both performances and physical achievements. In this paper some arguments presented at the RPSCINT-2013 conference are shortly summarized. More details, tables and figures can be found in the slides at the conference site [1].

\section{Requirements and realizations}

The used crystal growth technique can play a significant role in the crystal performances; we mention: i) the Bridgman-Stockbarger, which has the advantage to be the simplest method for growing alkali halide crystals; its main drawbacks are the fact that the crystal is in direct contact with crucible's walls, that stresses in growing crystal and extraction (cracks) are possible, that is difficult the uniform activator distribution through the ingot, that there is insufficient convectional melt mixing before the crystallization front (inclusions and striations), and that there is spontaneous crystallization on the ampoule surface (i.e. the orientation of the crystal is difficult to control), that a well-oriented seed is needed; ii) Czochralski, where the crystal is pulled from the melt; the seed crystal and crucible are rotated in opposite directions while withdrawal occurs; thus the crystal is not in direct contact with crucible's walls. This method allows one to increase in crystal growth rate owing to higher axial and radial temperature gradients and to intensive mixing of the melt by the rotating crystal. It seems to be very efficient when crystals with high structural perfection are required, however it is more technically complicated and permanent control and correction of the main parameters is needed. Thus, if there is non-automated pulling, the success of the growth 
is defined mainly by the skill of the operator; iii) Kyropoulos where the direct crystalization of the melt is obtained by decreasing the boule temperature while it is still in the crucible; in particular, crystal is not in direct contact with crucible's walls. Highly controlled thermal gradient keeps a low-stress environment for the crystal, and a deep control of all the parameters is needed. In addition, implementations of Czochralski-Kyropoulos techniques are operative and in evolution.

There are many topics which are important to realize ULB (Ultra-Low-Background) crystal scintillators besides the deep selection of the growing materials and tools; thus the creation of specific growing/handling protocols is mandatory. Among those topics we recall as examples: i) the role of the crucible material and of its cleaning procedure; ii) the deep selection of raw materials and their additional chemical/physical purification processes; iii) their handling, the quality of the nitrogen and the exclusivity of its supply line if the crystal is grown in nitrogen atmosphere; iv) the kind, the quality and the amount of the additives (always under confidential restriction in each company) used to improve the crystal performances; v) the relevance to avoid to open the furnace for checking during growth; vi) the tools and their cleaning procedures to handle and cut the crystal for a detector from the grown ingot, vii) the environment in the site of growing, cutting, handling, assembling the crystals. Moreover, it is worth noting that the growing procedures can act as a further purification step; in particular, in the Kyropoulos method in platinum crucible with special protocols it is a very effective one. In addition, the part of the ingot which is cut and used to produce the crystal scintillator is very important too, since the residual trace contaminants are generally distributed within the ingot depending - in a particular way - on the adopted growing procedures. Obviously, whatever else material (glue, optical windows, housing, etc.) entering in the assembling of the detector from the bare crystal should be selected with the same stringent requirements and treated with stringent protocol as well. A particular mention should be made about the abrasive tools selected to treat the crystal surface, since they could contaminate (obviously in a different way for each crystal) the surface of the crystal; for this reason, a deep selection of the abrasive tools to be used is necessary. Generally a compromise between light response and necessary surface cleanness is mandatory. In case of hygroscopic scintillators a very good choice for the housing is to use OFHC freshly electrolized copper; in this case, for some materials (as e.g. $\mathrm{NaI}(\mathrm{Tl})$ ) there is the necessity to avoid presence of the white mousse typically adopted to assure compensation of the different heat capacity of the crystal and of the housing materials and thus a peculiar design of the housing is necessary to avoid any possibility of break possibly due to large temperature variation during the detectors transportation at sea level.

Moreover, high-purity metals are necessary not only for applications in atomic energy production, microelectronics, space engineering, medicine and fundamental science researches, but also to realize some ULB crystal scin- tillators as e.g. $\mathrm{CdWO}_{4}$ and $\mathrm{ZnWO}_{4}$. There are several constraints which restrain the deep purification of metals: i) peculiarities of the behaviour of the impurities in the metal being purified, their mutual interactions and interactions with the base material; ii) infusion of impurities from structural materials in the processes of purification. One of the most radical ways of enhancing the efficiency of metal purification is to employ consecutively a number of refinement techniques with different mechanisms of impurity separation. In this way, a more effective separation of different classes of impurities is to be expected in comparison when only one technique, although very effective, is used many times.

As mentioned, the crystal scintillators show very interesting features, however, one should always remind that: i) the detectors are just one component of a low background experiment; ii) each detector has its own features; iii) each ULB (Ultra-Low-Background) production cannot exactly be reproduced e.g. because of: a) change of sources of materials; b) unavoidability of materials from the same selection; c) possible activation/pollution in (long) storage at sea level; d) different additives, different seeds; e) different procedures due to time modification of the production equipments, and of the safety rules; f) loss of some competences in the periodical change of involved people; g) etc. Moreover, the highest is the initial radio-purity the most difficult is to avoid to lose it in the handling in the company, in the packing, in the handling in the experiment, etc.

\section{Typical radioactive contaminants}

The main sources of radioactive contamination in scintillation materials are naturally occurring radionuclides of ${ }^{232} \mathrm{Th},{ }^{238} \mathrm{U}$, and ${ }^{235} \mathrm{U}$ families, and ${ }^{40} \mathrm{~K}$. In particular: i) the secular equilibrium of U/Th chains is generally broken in scintillation materials; ii) alpha active ${ }^{147} \mathrm{Sm}$ was detected in some scintillators at $\mathrm{mBq} / \mathrm{kg}$ level; iii) antropogenic ${ }^{60} \mathrm{Co},{ }^{90} \mathrm{Sr}-{ }^{90} \mathrm{Y},{ }^{137} \mathrm{Cs}$ nuclides may be present. Moreover, some scintillation crystals consist of elements having radioactive isotopes (as e.g.: ${ }^{152} \mathrm{Gd}$ in $\mathrm{GSO},{ }^{113} \mathrm{Cd}$ in $\mathrm{CdWO}_{4},{ }^{138} \mathrm{La}$ in $\mathrm{LaCl}_{3}$ and $\mathrm{LaBr}_{3},{ }^{176} \mathrm{Lu}$ in $\mathrm{Lu}_{2} \mathrm{SiO}_{5}$ and $\mathrm{LuI}_{3}$ ), and cosmogenic radionuclides, i.e. created by high energy cosmic rays or/and by neutrons, were also observed in some scintillation materials: ${ }^{14} \mathrm{C}$ in liquid scintillator, ${ }^{65} \mathrm{Zn}$ in $\mathrm{ZnWO}_{4},{ }^{152} \mathrm{Eu}$ in $\mathrm{CaF}_{2}(\mathrm{Eu}),{ }^{113 m} \mathrm{Cd}$ and ${ }^{110 m} \mathrm{Ag}$ in $\mathrm{CdWO}_{4},{ }^{207} \mathrm{Bi}$ in $\mathrm{BGO}$ whose origin is still not clear.

In Table 1 the radio-purity achieved in various crystal scintillators is reported.

HPGe detectors placed underground, high sensitive ICP-MS, AAS, etc, are used to select materials and estimate residual contaminants; however, the highest sensitivity to measure internal contamination of crystal scintillators is achieved in low background measurements where a scintillator is operating as a detector, by exploiting e.g. of: i) time-amplitude analysis; ii) pulse-shape discrimination, which e.g. in $\mathrm{NaI}(\mathrm{Tl})$ has a $100 \%$ discrimination power at the energies of the $\alpha$ particles; iii) energy spectra analysis (see for example Ref. [22]). 
Table 1. Radioactive contamination of some inorganic crystal scintillators $(\mathrm{mBq} / \mathrm{kg})$.

\begin{tabular}{|c|c|c|c|c|c|c|}
\hline Scintillator & ${ }^{228} \mathrm{Th}$ & ${ }^{226} \mathrm{Ra}$ & ${ }^{40} \mathrm{~K}$ & $\alpha$ activity & other radioisotopes & Ref. \\
\hline $\mathrm{NaI}(\mathrm{Tl})$ & $\begin{array}{l}0.002 \\
0.014 \\
0.02 \\
\end{array}$ & $\begin{array}{l}0.009 \\
0.045 \\
0.2 \\
\end{array}$ & $<0.6$ & $\begin{array}{l}0.08 \\
1.7 \\
\end{array}$ & & $\begin{array}{l}{[2]} \\
{[3]} \\
{[4]}\end{array}$ \\
\hline $\mathrm{CsI}(\mathrm{Tl})$ & 0.002 & $0.008^{*}$ & & & $\begin{array}{l}{ }^{134} \mathrm{Cs}(\simeq 14) \\
{ }^{137} \mathrm{Cs}(\simeq 6)\end{array}$ & {$[5]$} \\
\hline $\mathrm{ZnWO}_{4}$ & $0.002-0.018$ & $0.002-0.025$ & $\leq(0.01-1)$ & $0.2-2$ & ${ }^{65} \mathrm{Zn}(0.5-1)$ & {$[6]$} \\
\hline $\mathrm{CaWO}_{4}$ & 0.6 & 6 & $\leq 12$ & $20-400$ & $\begin{array}{l}{ }^{90} \mathrm{Sr}(\leq 70) \\
{ }^{137} \mathrm{Cs}(\leq 0.8) \\
{ }^{147} \mathrm{Sm}(0.5) \\
{ }^{180} \mathrm{~W}(0.05)\end{array}$ & [7] \\
\hline $\mathrm{CdWO}_{4}$ & $\leq 0.004-0.04$ & $\leq(0.004-0.04)$ & 0.3 & $0.3-2$ & $\begin{array}{l}{ }^{90} \mathrm{Sr}(\leq 0.2) \\
{ }^{113} \mathrm{Cd}(558) \\
{ }^{137} \mathrm{Cs}(\leq 0.3-0.4) \\
{ }^{147} \mathrm{Sm}(\leq 0.01-0.04) \\
{ }^{180} \mathrm{~W}(0.04)\end{array}$ & {$[8-10]$} \\
\hline $\begin{array}{l}\mathrm{PbWO}_{4} \\
\mathrm{PbWO}_{4} \\
\text { (ancient } \mathrm{Pb} \text { ) }\end{array}$ & $\leq 13$ & $\leq 10$ & & $(53-79) \cdot 10^{3}$ & $\begin{array}{l}{ }^{210} \mathrm{~Pb}\left((53-79) \cdot 10^{3}\right) \\
{ }^{210} \mathrm{~Pb}(\leq 4)\end{array}$ & $\begin{array}{l}{[11]} \\
{[12]}\end{array}$ \\
\hline $\mathrm{CaMoO}_{4}$ & 0.04 & 0.13 & $\leq 3$ & $\leq 10$ & & {$[13]$} \\
\hline $\mathrm{CaF}_{2}(\mathrm{Eu})$ & $\begin{array}{l}0.13 \\
0.1 \\
\end{array}$ & $\begin{array}{l}1.3 \\
1.1 \\
\end{array}$ & $\leq 7$ & 8 & ${ }^{152} \mathrm{Eu}(10)$ & $\begin{array}{l}{[14]} \\
{[15]}\end{array}$ \\
\hline $\mathrm{CeF}_{3}$ & 1100 & $\leq 60$ & $\leq 330$ & 3400 & & {$[16]$} \\
\hline $\mathrm{BaF}_{2}$ & 400 & 1400 & & & & {$[17]$} \\
\hline $\mathrm{BGO}$ & $<0.4$ & $<1.2$ & & & ${ }^{207} \mathrm{Bi}\left(7-3 \cdot 10^{3}\right)$ & {$[18,19]$} \\
\hline $\mathrm{LaCl}_{3}(\mathrm{Ce})$ & $\leq 0.4$ & $\leq 34$ & 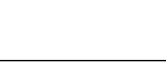 & & ${ }^{138} \mathrm{La}\left(21 \cdot 10^{3}\right)$ & [20] \\
\hline
\end{tabular}

A good energy resolution is useful in particular in the $\beta \beta$ decay investigations since it facilitates the separation of the $0 v \beta \beta$ decay mode from the $2 v \beta \beta$ one and to separate a $0 v \beta \beta$ signal from other $\gamma$ lines. However, relatively modest energy resolution can be acceptable for most decay modes and, in particular: i) to separate $0 v \beta \beta$ from $2 v \beta \beta$ when fitting the end-point shape (procedure adopted e.g. in NEMO-3); ii) to separate a $0 v \beta \beta$ signal from other $\gamma$ lines in case of low-level background by choosing a high Q-value isotope and/or by using an ultra-low background detector and set-up; thus, a distinctive peak can be pointed out from a low-level continuum background.

\section{Features and capabilities}

Let us recall the main crystal scintillator characteristics: i) the Stokes shift, i.e. the difference - in wavelength or frequency units - between the positions of the band maxima of the absorption and emission spectra of the same electronic transition; ii) the light yield; iii) the emission spectrum; iv) the decay time; v) the density; vi) the $\mathrm{Z}$ value; vii) the afterglow (phosphorescence after some $\mathrm{ms}$ ), caused by impurities or defects that create traps or metastable states with long lifetime (in low background measurements with pure crystals or e.g. $\mathrm{NaI}(\mathrm{Tl})$ blocking time can be used in the DAQ as well as rejection by 
PSD being well different the decay time of the scintillation pulse and of the afterglow single photoelectrons).

Some inorganic crystals show some temperature dependence of the light output; for example in $\mathrm{NaI}(\mathrm{Tl})$ variation with slope of the light output:- $0.2 \% /{ }^{\circ} \mathrm{C}$ is typically observed. In low background experiment very high temperature stability can be assured by continuous air conditioning and by direct contact between the metallic housing of the detectors with a multi-ton metallic shield which can assure a huge overall heat capacity $\left(\approx 10^{6} \mathrm{cal} /{ }^{\circ} \mathrm{C}\right)$ and a high temperature stability of the crystals (see e.g. in ref. [2]). Moreover, regular routine calibrations at suitable intervals allow one to avoid any significant systematic uncertainties in whatever kind of detector and operating conditions. It is worth noting that instead even a very modest temperature variation would limit - with systematic uncertainty - the sensitivity of pulse shape discrimination at very low energy where the discrimination capability of inorganic crystals becomes marginal.

A significant role is played by the PMTs coupled to the scintillators, and in particular - among others - by the transmission of PMT window and by the quantum efficiency (Q.E.). As regards the PMT window we remind that $\mathrm{NaF}, \mathrm{MgF}_{2}, \mathrm{LiF}$ and $\mathrm{CaF}_{2}$ are window materials necessary to collect scintillation light with $\lambda$ below $150 \mathrm{~nm}$, while fused silica can be used effectively above $\simeq 180 \mathrm{~nm}$, UV glass above $220 \mathrm{~nm}$, borosilicate glass above $280 \mathrm{~nm}$, etc. As regards the quantum efficiency, recent developments such as e.g. the one in ref. [23] have allowed to significantly improve it; in particular, there the following value, averaged over the 50 PMTs, has been measured at peak: $38.5 \%$ (1.6\% RMS), with best value: 43.1 and worst value: $36 \%$.

\section{Activities}

A large number of isotopes are present in various kind of crystal scintillators and double beta decay modes can be effectively investigated with the source=detector approach. Moreover, the possibility to create detectors enriched in some specific isotopes is another very interesting feature.

Among the existing activities, I remind here the one carried out by the DAMA-Kiev collaboration which has already developed various kinds of low background scintillators and carried out many measurements mainly profiting of the low background DAMA/R\&D set-up, such as e.g. $\mathrm{CaF}_{2}(\mathrm{Eu}), \mathrm{CeF}_{3}, \mathrm{BaF}_{2}, \mathrm{CdWO}_{4},{ }^{106} \mathrm{CdWO}_{4}$, ${ }^{116} \mathrm{CdWO}_{4}, \mathrm{ZnWO}_{4}, \mathrm{LaCl}_{3}(\mathrm{Ce}), \mathrm{LiEu}\left(\mathrm{BO}_{3}\right)_{3}, \mathrm{LiI}(\mathrm{Eu})$, $\mathrm{LiF}(\mathrm{W}), \mathrm{CeCl}_{3}, \mathrm{Li}_{2} \mathrm{MoO}_{4}, \mathrm{SrI}_{2}(\mathrm{Eu})$ [24-31]. In particular, enriched scintillators can offer an unique possibility to investigate in relatively small set-ups the $\beta \beta$ decay modes in various isotopes with high sensitivity.

Moreover, highly radio-pure $\mathrm{NaI}(\mathrm{Tl})$ crystal scintillators have been successfully developed by DAMA in collaboration with leader company and many competing results have been and can be achieved [2].

\section{Conclusion}

The effectiveness of low background scintillation technique to search for rare processes has been proved and the pursuing of continue efforts to develop new/improved crystal scintillators for low background physics is a very fruitful strategy.

Large efforts in this field are carried out by the DAMAKiev collaboration that investigates many rare processes with high sensitivity using the facility DAMA/R\&D, as well as widely DAMA/Ge and LNGS STELLA facility. In particular the experiments on $2 \beta$ decay of ${ }^{106} \mathrm{Cd}$ and ${ }^{116} \mathrm{Cd}$ are running/under-improvement and other new measurements are in preparation and/or foreseen (using isotopes of $\mathrm{Ce}, \mathrm{Ba}, \mathrm{Gd}, \mathrm{Nd}, \mathrm{Ru}, \mathrm{Sr}$, etc.); a new small facility, named DAMA/CRYS, is starting the activity.

Moreover, the second generation DAMA/LIBRA setup by DAMA coll. is running in its improved phase 2 configuration with new higher quantum efficiency PMTs.

\section{References}

[1] R. Bernabei, presentation in this Conference: http://medex13.utef.cvut.cz/talks/Bernabei.pdf

[2] R. Bernabei et al., Nucl. Instr. \& Meth. A 592, 297 (2008); R. Bernabei et al., Eur. Phys. J. C 56, 333 (2008); R. Bernabei et al., Eur. Phys. J. C 67, 39 (2010); R. Bernabei et al., Int. J. of Mod. Phys. A 28, 1330022 (2013); R. Bernabei et al., Eur. Phys. J. C 73, 2648 (2013); and refs. therein

[3] J.C. Barton, J.A. Edgington, Nucl. Instr. Meth. A 443, 277 (2000)

[4] J. Amaré et al., J. Phys.: Conf. Series 39, 201 (2006)

[5] H.S. Lee et al., Nucl. Instr. \& Meth. A 571, 644 (2007); S.S. Myung et al., AIP Conf. Proc. 1078, 533 (2009)

[6] P. Belli et al., Nucl. Instr. \& Meth. A 626-627, 31 (2011); Acta Phys. Polonica A 117, 139 (2010); Nucl. Phys. A 826, 256 (2009); R. Bernabei et al., Phys. Lett. B 658, 193 (2008); J. Phys. G: Nucl. Part. Phys. 38, 115107 (2011)

[7] Yu.G. Zdesenko et al., Nucl. Instr. \& Meth. A 538, 657 (2005); C. Cozzini et al., Phys. Rev. C 70, 064606 (2004)

[8] P. Belli et al., Phys. Rev. C 76, 064603 (2007)

[9] F.A. Danevich et al., AIP Conf. Proc. 785, 87 (2005)

[10] F.A. Danevich et al, AIP Conf. Proc. 1549, 201 (2013)

[11] F.A. Danevich et al., Nucl. Instr. \& Meth. A 556, 259 (2206)

[12] A. Alessandrello et al., Nucl. Instr. \& Meth. A 409, 451(1998)

[13] A.N. Annenkov et al., Nucl. Instr. \& Meth. A 584, 334 (2008)

[14] R. Bernabei et al., Nucl. Phys. A 705, 29 (2002); P. Belli et al., Nucl. Phys. B 563, 97 (1999); R. Bernabei et al., Astropart. Phys. 7, 73 (1999)

[15] I. Ogawa et al., Nucl. Phys. A 730, 215 (2004); S. Umehara et al., Phys. Rev. C 78, 058501 (2008) R. 
Hazama et al., WEIN 95, p. 635 in: Proc. of the 4th Int. Conf. on Weak and Electromagnetic Interactions in Nuclei, Osaka, Japan, June 1995, World Scientific, Singapore, 1995

[16] P. Belli et al., Nucl. Instr. \& Meth. A 498, 352 (2003)

[17] R. Cerulli et al., Nucl. Instr. \& Meth. A 525, 535 (2004)

[18] A. Balysh et al., Pribory i Tekhnika Eksperimenta 1, 118 (1993) (in Russian)

[19] P. de Marcillac et al., Nature 422, 876 (2003)

[20] R. Bernabei et al., Nucl. Instr. \& Meth. A 555, 270 (2005)

[21] J. Lee, slides of presentation given at PPC2012 Conference, KIAS, Korea, 2012

[22] P. Belli et al., Phys. Rev. C 85, 044610 (2012)

[23] R. Bernabei et al., J. Instr. 7, P03009 (2012)

[24] R. Bernabei et al., Astropart. Phys. 7, 73 (1997); Nuovo Cim. A 110, 189 (1997); P. Belli et al., Astropart. Phys. 10, 115 (1999); Nucl. Phys. B 563, 97
(1999); R. Bernabei et al., Nucl. Phys. A 705, 29 (2002); P. Belli et al., Nucl. Instr. Meth. A 498, 352 (2003); R. Cerulli et al., Nucl. Instr. Meth. A 525, 535 (2004); R. Bernabei et al., Nucl. Instr. Meth. A 555, 270 (2005); Ukr. J. Phys. 51, 1037 (2006); P. Belli et al., Nucl. Phys. A 789, 15 (2007); Phys. Rev. C 76, 064603 (2007); Eur. Phys. J. A 36, 167 (2008); Nucl. Instr. Meth. A 615, 301 (2010); J. Phys. G: Nucl. Part. Phys. 38, 015107 (2011); A.S. Barabash et al., J. Instr. 6, P08011 (2011)

[25] P. Belli et al., Phys. Rev. C 85, 044610 (2012)

[26] P. Belli et al., Phys. Lett. B 711, 41 (2012)

[27] P. Belli et al., Nucl. Instr. Meth. A 670, 10 (2012)

[28] P. Belli et al., Nucl. Instr. Meth. A 626-627, 31 (2011); P. Belli et al., Nucl. Phys. A 826, 256 (2009); P. Belli et al., Phys. Lett. B 658, 193 (2008)

[29] O.P. Barinova et al., Nucl. Instr. Meth. A 607, 573 (2009)

[30] P. Belli et al., Nucl. Instr. Meth. A 572, 734 (2007)

[31] P. Belli et al., Nucl. Instr. Meth. A 704, 40 (2013) 